Revue d'histoire de l'enfance « irrégulière »

Le Temps de l'histoire

Hors-série | 2007

Pages d'histoire, la protection judiciaire des mineurs, $\mathrm{XIX} \mathrm{X}^{\mathrm{e}} \mathrm{XX} \mathrm{X}^{\mathrm{e}}$ siècles

\title{
Un statut qui précède le métier
}

L'éducateur de l'Éducation surveillée et le statut du 10 avril 1945

Jacques Bourquin

\section{(2) OpenEdition}

Journals

Édition électronique

URL : http://journals.openedition.org/rhei/3009

DOI : $10.4000 /$ rhei.3009

ISBN : 978-2-7535-1647-2

ISSN : 1777-540X

Éditeur

Presses universitaires de Rennes

Édition imprimée

Date de publication : 1 juin 2007

Pagination : 97-106

ISSN : 1287-2431

\section{Référence électronique}

Jacques Bourquin, «Un statut qui précède le métier », Revue d'histoire de l'enfance « irrégulière » [En

ligne], Hors-série | 2007, mis en ligne le 01 février 2010, consulté le 30 avril 2019. URL : http:// journals.openedition.org/rhei/3009; DOI : 10.4000/rhei.3009 


\section{Un statut qui précède le métier L'éducateur de l'Éducation surveillée et le statut du 10 avril $1945^{(*)}$}

\section{Introduction}

Les premiers éducateurs du secteur de l'enfance inadaptée et les premières écoles pour les former apparaissent en 1942-1943. Il faudra près de 25 ans pour que ce métier bénéficie d'une véritable reconnaissance officielle concrétisée par un diplôme d'État.

Il en avait été de même pour les assistantes de service social apparues, ainsi que les premières écoles, dès le début du siècle, alors que le statut n'interviendra que trente ans plus tard en 1932.

Avec les éducateurs de l'Éducation surveillée, ceux du ministère de la Justice, il en sera tout autrement : nous sommes dans la fonction publique; c'est le statut qui précède le métier et les organismes de formation.

Si le métier d'éducateur est récent, à peine un demi-siècle, on en parle depuis bien plus longtemps, en particulier dans le domaine des enfants de Justice où il a pris racine.

F.-A. Demetz, fondateur en 1839 de la colonie agricole et pénitentiaire de Mettray, lui avait donné une sorte d'ancêtre avec l'institution des chefs de famille chargés d'encadrer les colons; il mit même en place pour ces nouveaux agents une école de formation, l'école des contremaîtres qui dura une trentaine d'années. La colonie de Mettray évolua de telle manière que, vers la fin du siècle, le chef de famille, dans un univers de plus en plus répressif, était devenu un véritable gardien.

Une loi de 1850 sur l'éducation et le patronage des jeunes détenus prévoyait la création par la direction de l'Administration pénitentiaire d'établissements spécialisés pour mineurs : les colonies correctionnelles et pénitentiaires. Il n'était rien prévu quant à la spécialisation des personnels, qui pouvaient aussi bien être affectés dans des établissements pour majeurs que pour
(*) Article publié

dans le Temps de l'histoire,

n 1 , février 1998.

Ce texte est la communication de l'auteur aux journées d'études l'Éducation surveillée aurait eu so ans, Vaucresson, les 9 et 10 novembre 1995 .

Un statut qui précède le métier... / p. 97 à 106 
(1) L'enfant à travers les âges. Section de l'éducation pénitentiaire, Imp. adm. de Melun, mai 1901, p. 11.

(2) Paul Cuche, Traité de science et de législation pénitentiaires, Paris, librairie générale de droit et de jurisprudence, 1905. mineurs. On était surtout dans une logique de garde, de discipline, de châtiment, où la discipline était devenue progressivement l'unique élément de moralisation, où le travail avait perdu sa fonction d'insertion sociale et d'éducation pour ne plus être qu'un simple élément de la peine.

Il faut attendre la fin du XIXème siècle pour qu'apparaisse un courant de réforme pénitentiaire, influencé par l'École de défense sociale, qui privilégie l'individualisation de la peine et la prise en compte de la personnalité du délinquant. Des comités de défense des enfants traduits en justice composés de juristes, de médecins, de philanthropes seront les acteurs de ce mouvement de réforme, en particulier sur le plan législatif. Au congrès international pénitentiaire de Bruxelles, en août 1900, la direction de l'Administration pénitentiaire française évoque, dans son rapport, le projet de substitution de maisons d'éducation pénitentiaire aux colonies pénitentiaires et correctionnelles. "L'idée de répression, précise le rapport, a fait place au principe plus humain de l'éducation "; ${ }^{(1)}$ on assouplit le régime disciplinaire

Le congrès fait plusieurs fois référence aux " éducateurs ", mais ce terme recouvre en fait les "surveillants ", nouvelle appellation des gardiens depuis 1894, "qui doivent jouer un rôle important dans l'éducation morale des pupilles ». On évoque aussi les " instituteurs » (ils sont 3 pour 300 colons à la colonie de Lamotte-Beuvron), qui « donnent un enseignement scolaire élémentaire..., qui doivent fournir au directeur une étude psychologique sur les enfants qu'ils ont à instruire et moraliser ". Plus que la notion d'éducation, c'est celle d'instruction qui est évoquée. Le rapport conclut au fait qu' " être éducateur n'est pas un métier, ni une fonction, c'est une grande mission morale et sociale ". Quant au doyen Cuche, ${ }^{(2)}$ pénaliste distingué qui s’intéresse à la réforme pénitentiaire, il se demande si la solution ne consisterait pas à placer auprès de chaque pupille un " ange gardien », image pleine de religiosité. Mais, dans l'optique pénitentiaire, le gardien peut vite se substituer à l'ange.

La loi du 22 juillet 1912 sur l'enfance délinquante, malgré ses ouvertures, demeure très théorique et particulièrement silencieuse en ce qui concerne les institutions pour mineurs. Elle provoque toutefois, au lendemain de la guerre de 1914, un recours de moins en moins important aux colonies pénitentiaires et correctionnelles, les tribunaux choisissant de plus en plus les placements dans les patronages, apparus vers 1890 dans le domaine de l'enfance 
irrégulière. L'éducateur reste inexistant; les mineurs sont le plus souvent placés dans des familles rurales sous le contrôle des sociétés de patronage. Jean Pinatel ${ }^{(3)}$ parlera à cette occasion de "la déflation pénitentiaire qui consacre l'incurie de l'Administration pénitentiaire à s'occuper des mineurs ».

\section{Du moniteur à l'éducateur de l'Éducation surveillée (1927-1945)}

Alors que l'inspecteur général Mosse, ${ }^{(4)}$ dans un rapport de 1927, propose que l'on dissocie à l'Administration pénitentiaire le cadre des personnels s'occupant des majeurs de celui des mineurs, un décret du 31 décembre 1927, créant un service de l'Éducation surveillée à l'intérieur de l'Administration pénitentiaire, consacre l'uniformité du recrutement et l'interchangeabilité du personnel des services de majeurs et de mineurs. Le décret se contente de modifier les appellations : " les colonies correctionnelles " deviennent " maisons d'éducation surveillée ». Les " surveillants " prennent le nom de " moniteurs », mais leur fonction ne change nullement. Quant aux colons, ils s'appelleront dorénavant " pupilles ». On a parlé à l'époque de "réforme sur le papier ».

Un règlement du 15 février 1930, en s'efforçant de concrétiser la notion de l'Éducation surveillée, distingue :

- le personnel administratif et éducatif, corps commun, où l'instituteur peut accéder aux fonctions de direction, mais il lui faut pour cela passer par les grades de greffier comptable, d'économe et de sous-directeur. Sa vocation pédagogique est progressivement niée au profit d'une fonction gestionnaire : en témoigne cette appréciation de 1936 : «Monsieur X est un bon instituteur, il rend déjà d'appréciables services à l'économat ". À ce corps sont adjoints deux autres corps :

- les personnels de surveillance : moniteur, maître et premier maître.

- les personnels techniques : sous-chef d'atelier, chef d'atelier, ingénieur.

Il faut attendre un décret du 26 octobre 1935 pour que, dans le contexte des campagnes de presse contre les bagnes d'enfants qui ont débuté en 1934, on prévoit que le tiers des emplois vacants des services de l'Éducation surveillée soit réservé aux candidats possédant le diplôme tout récent d'assistant social ou le certificat d'aptitude à l'enseignement des enfants arriérés prévu par une loi de 1909. Ce décret n'aura aucune application, car il n'y aura aucun candidat.
(3) Jean Pinatel,

"Le statut de l'éducateur ", Revue de l'Éducation surveillée, $\mathrm{n}^{\circ} 1$, 1946, p. 52-63.

(4) Armand Mosse, Rapport de l'Administration pénitentiaire, Imp. adm. de Melun, 1927. 
(5) Entretien de

Michel Chauvière avec Jacques Guérin-

Desjardins, en 1980.

(6) Alexis Danan,

journaliste qui fut l'animateur des campagnes de presse contre les bagnes d'enfants (1934-1937).

(7) À ce sujet, lire Henri Gaillac, Les maisons de correction, Paris, Éditions Cujas, 1972, p. 305-311.

\section{Les premiers décrets de réforme, 1936-1938}

Le 13 août 1936, le Garde des sceaux, Marc Rucart fait voter la réforme de la maison d'éducation surveillée de Saint-Maurice à Lamotte-Beuvron. Cette loi prévoit que le personnel éducateur ne sera plus recruté directement parmi les personnels de l'Administration pénitentiaire. Il sera détaché de l'Éducation nationale et de la direction de l'Enseignement technique qui dépend à l'époque du ministère du Travail. On parle de " moniteurs-éducateurs ».

Quelques instituteurs volontaires ou désignés par le ministère de l'Éducation nationale, environ une quinzaine, suivront un stage de formation d'un mois à la prison de Fresnes. L'organisation en a été confiée à Jacques GuérinDesjardins, ${ }^{(5)}$ commissaire général des Éclaireurs, qui dira de ces stagiaires : " Ils sont peu motivés, à l'exception de 5 ou 6 qui sortent du scoutisme ou de la JOC. Ils sont surtout très excités par les campagnes de presse menées par Alexis Danan. ${ }^{(6)}$ La presse de l'époque est dithyrambique à l'égard de ces jeunes moniteurs-éducateurs : "On prend soin d'équiper ces héros en vue de la tâche difficile qu'ils auront à assumer " (Paris Soir, 10 janvier 1937). Leur rencontre avec la maison d'éducation surveillée de Saint-Maurice à Lamotte-Beuvron se révélera un échec au bout de quelques semaines. Ils n'étaient guère attendus par les personnels en place et guère plus préparés à leur nouvelle mission. ${ }^{(7)}$

Il faudra attendre avril 1938 pour qu'une nouvelle tentative de réforme soit engagée à la maison d'éducation surveillée de Saint-Maurice et celle de Saint-Hilaire.

À Saint-Maurice, la direction de l'Administration pénitentiaire fait appel à la direction de l'Enseignement technique, et c'est sur le modèle de la formation professionnelle que s'élaborera une réforme qui va réussir.

Ces deux tentatives éducatives vont être officialisées par un décret du 17 août 1938 qui envisage enfin un cadre distinct entre les personnels s'occupant de mineurs et ceux s'occupant de majeurs. C'est la fin de l'interpénétration entre les deux corps. Le vœu de l'inspecteur général Mosse est réalisé.

Le même mois (août 1938), Jean Doublet, auditeur au Conseil d'État, rédige un projet de statut des éducateurs de l'Éducation surveillée : les conditions de recrutement sont celles des instituteurs non normaliens (bac ou brevet supérieur ou certificat d'aptitude pédagogique).

On envisage aussi que les éducateurs puissent être recrutés, après examen 
d'aptitude et avis d'une commission, parmi les instituteurs et moniteurséducateurs de l'Administration pénitentiaire, ainsi que parmi les instituteurs de l'Éducation nationale. Les emplois de sous-directeur et directeur seront exclusivement réservés aux éducateurs. Le projet prévoit ce que pourraient être leurs obligations professionnelles :

« la surveillance, l'éducation physique... Ils veillent avec le surveillant général à la discipline de tous les instants... Ils ont la charge de l'enseignement scolaire, des veillées causeries et des correspondances des pupilles. "

"Les éducateurs stagiaires ne seront titularisés qu'au bout de 2 ans minimum, ils rempliront pendant la première année de leur stage les fonctions de moniteurs ", c'est-à-dire de surveillants.

Il est à remarquer qu'on ne prévoit aucune formation spécifique, et on peut penser que ces jeunes éducateurs auront vraisemblablement bien du mal, dans un contexte où la surveillance et la discipline sont majorées, pour trouver leur véritable fonction auprès des jeunes.

Le texte qui s'inscrit parfaitement dans les tentatives de réforme de 1938 à Saint-Maurice et à Saint-Hilaire restera sans lendemain.

\section{Le décret du 31 août 1940, un texte paradoxal}

Ce décret, en créant les institutions publiques d'éducation surveillée (IPES) qui se substituent aux maisons d'éducation surveillée de 1927, semble inaugurer une période centrée sur la rééducation et la formation professionnelle. Il définit l'IPES comme une institution qui doit donner " une éducation morale, religieuse et professionnelle " et où " le degré d'enseignement doit correspondre à celui des écoles de métiers et des écoles d'agriculture ". Paradoxalement, ce même décret réintroduit l'interpénétration entre les deux cadres de personnel, pénitentiaire et d'Éducation surveillée. La conséquence en est que, jusqu'à fin 1942, l'Administration pénitentiaire ne recrutera plus dans ses établissements pour mineurs que des " moniteurs ", soit un personnel de surveillance.

Quelle justification peut-on donner à cette situation qui apparaît comme une véritable mise en sommeil de la volonté de réforme?

Vraisemblablement, mais cela mériterait d'être vérifié sur le plan statistique, l'augmentation massive des mineurs placés dans les établissements de 
(8) Jean Bancal, Essai sur le redressement de l'enfance coupable, Paris, Recueil Sirey, 1941.

(9) Jacques Bourquin,

«Sur les traces des premiers éducateurs de l'Éducation surveillée, 1936-1947 ", Cabiers du $C R I V, \mathrm{n}^{\circ} 2$, Vaucresson, octobre 1986.
l'Éducation surveillée. Suite à la fragilisation de bien des familles liée à l'exode, à l'absence de plus d'un million de pères prisonniers en Allemagne et aux difficultés créées par l'occupation, on assiste, à partir de 1940, à une recrudescence de la délinquance juvénile.

En 1941, l'inspecteur général Jean Bancal publie un rapport ${ }^{(8)}$ dans lequel il propose la suppression de la hiérarchie traditionnelle du personnel pénitentiaire pour la remplacer par trois cadres :

- un cadre administratif, où est maintenu le directeur;

- un cadre d'éducation;

- un cadre technique (formation professionnelle).

On retrouve là des éléments du projet de 1938, en particulier la spécialisation du personnel d'éducation, dont on ne sait cependant ce qu'il sera réellement. Bancal maintient le directeur dans le cadre administratif contrairement au projet de 1938, mais il souhaite qu'il privilégie sa fonction éducative et qu'il soit mieux secondé dans ses tâches administratives.

Ce projet Bancal, qui préfigure le statut des personnels de l'Éducation surveillée du 10 avril 1945, n'aura pas d'application pendant la période de Vichy. Toutefois à partir de fin 1942, le service de l'Éducation surveillée et son responsable Pierre Ceccaldi accueilleront le plus souvent comme " moniteur ", plus rarement " moniteur-éducateur », des jeunes gens pour lesquels l'entrée à l'Éducation surveillée sera un moyen d'échapper au STO (Service du travail obligatoire en Allemagne). Ce sont ces personnels souvent étudiants, chômeurs, instituteurs qui introduiront en particulier à Saint-Hilaire, à un degré moindre à Saint-Maurice, et dans les centres d'accueil ouverts dans la région parisienne, un espace éducatif fondé sur la vie en groupe, les loisirs, à partir de fréquentes références au scoutisme. (9)

À la même époque, dans le secteur privé où se développent les premiers centres d'accueil pour mineurs délinquants, apparaissent les premiers éducateurs et les premières écoles (Montpellier, Lyon, Montesson). Le service de l'Éducation surveillée, plus modestement, organisera, en novembre 1943, à l'université de Clermont-Ferrand, une session de 15 jours mise sur pied par un psychiatre, le Pr. Lagache, et un jeune magistrat de l'Éducation surveillée, Paul Lutz. Cette session, animée par le Pr. Daniel Lagache, privilégie la dimension psychologique de la délinquance, considérée « comme une inadaptation de 
l'individu à ses conflits ». On se réfere à la psychanalyse, on y parle de psychothérapie, de cure libre, mais aussi "d'éducation en groupe en internat", en insistant sur la notion de "self government " qui n'est pas sans lien avec la pédagogie scoute, très présente dans les premiers pas de ce qui deviendra l'éducation spécialisée. On sent, dans ce projet ambitieux, une volonté du modeste service de l'Éducation surveillée, toujours dépendant de l'Administration pénitentiaire, de tourner la page de la prise en charge restée surtout disciplinaire des mineurs délinquants.

\section{Le statut des personnels de l'Éducation surveillée du 10 avril 1945 : un aboutissement}

Le décret intervient deux mois après la promulgation de l'ordonnance sur l'enfance délinquante et quatre mois avant que l'Éducation surveillée, en devenant direction, coupe ses liens avec l'Administration pénitentiaire.

En établissant une différenciation absolue entre le cadre des personnels pénitentiaires et celui des personnels de l'Éducation surveillée, le décret affirme une véritable coupure institutionnelle entre les deux administrations, mais aussi l'identité éducative de la nouvelle institution. Concrètement, le décret prévoit la création d'une " commission de reclassement ", qui va avoir pour mission de répartir nominativement tous les fonctionnaires en activité soit dans les services extérieurs de l'Administration pénitentiaire, soit dans les services extérieurs de l'Éducation surveillée. Il n'y a donc plus, comme par le passé, d'interchangeabilité entre les deux corps. Toutefois, prévoyant que la nouvelle direction aura du mal à assurer, au cours des premières années, un recrutement extérieur, le texte envisage qu' " un fonctionnaire pourra être laissé provisoirement, pour une durée qui ne pourra excéder deux ans, dans un cadre autre que celui qui lui est affecté ". 160 personnels pénitentiaires seront ainsi maintenus provisoirement à l'Éducation surveillée, le plus souvent dans des fonctions d'agent de service. Ce maintien durera plus de deux ans, puisque les derniers personnels de ce type quitteront l'Éducation surveillée vers 1950.

Le statut prévoit trois cadres de personnels pour l'Éducation surveillée :

- un cadre de personnel éducateur; ${ }^{(10)}$

- un cadre de personnel administratif;

(10) À l'époque, on ne parle pas de " personnel éducatif ». 
(11) Certificat d'aptitude au professorat d'éducation physique.
- un cadre de personnel d'enseignement professionnel.

Comme dans le projet de 1938, le directeur appartient au cadre du personnel éducateur. Sont donc exclus des fonctions de directeur les personnels administratifs et d'enseignement professionnel. Il y a là un véritable pari quant à l'évolution de la fonction éducative encore si peu perceptible en 1945, alors que l'homme fort de l'internat de rééducation est à cette époque l'enseignant professionnel. L'exclusion des personnels administratifs des fonctions de direction peut apparaitre comme la volonté de ne pas reproduire à l'Éducation surveillée le modèle hiérarchique de l'Administration pénitentiaire, où tout directeur, même s'il a été instituteur au début de sa carrière, doit s'engager dans une filière administrative et gestionnaire (commis greffier, greffier, économe) pour accéder à sa fonction.

Le principe de recrutement des nouveaux personnels est celui du concours. Il est demandé aux futurs éducateurs d'être titulaires du baccalauréat ou du brevet supérieur pour l'emploi d'éducateur adjoint, premier grade de la fonction éducative. Larticle 8 du statut ajoute que « les candidats ayant obtenu des diplômes ou effectué des stages les préparant à leur mission éducative peuvent soit bénéficier de majoration de points, soit être admis à concourir en n'étant titulaires que du certificat d'études ou du brevet élémentaire ". Par ce biais, l'Éducation surveillée, à la recherche de candidats, réduit les exigences de diplôme, tout en essayant de recruter des personnels bacheliers ou non ayant bénéficié de la formation des écoles de cadres mises en place par le secteur privé en 1943-1944. Le décret du 10 avril 1945 ne prévoit aucune formation des personnels organisée par l'Éducation surveillée.

Dans son article 9, le statut prévoit que les titulaires d'un diplôme d'enseignement supérieur pourront être recrutés comme éducateurs-chefs; il y en aura quelques-uns et c'est parmi eux que seront inscrits les premiers psychologues des centres d'observation. Un sort particulier sera réservé aux professeurs d'éducation physique recrutés avec la deuxième partie du CAPEP. ${ }^{(11)}$ Dans la hiérarchie, ils seront entre le sous-directeur et le chef de service éducatif.

Il faudra attendre jusqu'en 1951 pour que la direction de l'Éducation surveillée ait les moyens financiers d'organiser le premier concours d'éducateurs. Entre temps, il s'agira surtout de recrutements locaux plus ou moins sur la base du statut. La règle étant, pour la direction de l'Éducation surveillée, de titula- 
riser au bout d'un an les éducateurs et éducateurs adjoints sur le rapport du directeur de l'institution. Ce recrutement s'étendra aussi :

- aux personnels pénitentiaires retenus définitivement par la commission de reclassement de novembre 1945 : 238 moniteurs, moniteurs-éducateurs, instructeurs, personnels administratifs seront intégrés dans la nouvelle direction. Nombre d'entre eux avaient participé aux réformes de 1938 ou étaient entrés à l'Éducation surveillée à partir de 1942. Il est à remarquer que ceux qui avaient été recrutés en 1942-1943 pour échapper au STO quitteront dans leur grande majorité l'Éducation surveillée, dès les lendemains de la Libération.

- aux instituteurs de l'Éducation nationale dont on sollicitera le détachement. Ils seront quelques dizaines à entrer à l'Éducation surveillée entre 1945 et 1947 , mais peu d'entre eux resteront.

La grande lacune du statut est l'absence totale de structure de formation des personnels. On se situe à ce niveau en retrait par rapport au projet Bancal de 1941, qui insistait fortement sur une spécialisation des personnels éducatifs. Il faudra attendre 1951 pour qu'ouvre, avec Henri Michard, le Centre d'études et de formation de Vaucresson. En attendant, auront lieu quelques sessions épisodiques de formation à Savigny-sur-Orge et à Marly-le-Roi, où deux personnels par institution viennent participer à un stage de deux semaines, dont ils retransmettront le contenu dans leur institution. Henri Michard, qui fut l'instigateur de ces premières réunions, parlait à l'époque de "formation missionnaire ". Que l'époque ait accordé de l'importance à une formation empirique, une formation sur le tas pour ce qui n'était même pas encore un métier, c'est vraisemblable. Pourtant, le projet de ce qui allait devenir le Centre de Vaucresson était déjà rédigé en 1945. On peut supposer que, pour la toute jeune direction de l'Éducation surveillée, qui dispose de faibles moyens, la priorité est donnée à la réforme des établissements et surtout à la formation professionnelle, fer de lance de la rééducation de 1945 et des années qui suivent. On a envie de dire, et c'est ce qui s'est partiellement passé : l'éducateur viendra après.

Pour la fonction publique, ce qui était essentiel, c'était d'inscrire la fonction d'éducateur dans un statut. Ce fut réalisé avant même que l'Éducation surveillée se sépare de l'Administration pénitentiaire.

Qu'en est-il pour ce futur éducateur? 
Il faut lui laisser le temps de se construire, de trouver ses contours, de s'autolégitimer dans un vécu avec les jeunes, en dehors des discours théoriques sur l'enfance délinquante.

L'éducateur va s'installer; l'avenir semble pour lui. Il a un statut qu'il va lui falloir défendre, il se syndique, il réclame une formation.

Cette autolégitimation, soutenue par le statut et la formation, lui fera prendre conscience progressivement de sa place auprès des jeunes dans l'institution. 